\title{
Prolyl isomerase Pin1 and protein kinase HIPK2 cooperate to promote cortical neurogenesis by suppressing Groucho/TLE:Hes1-mediated inhibition of neuronal differentiation
}

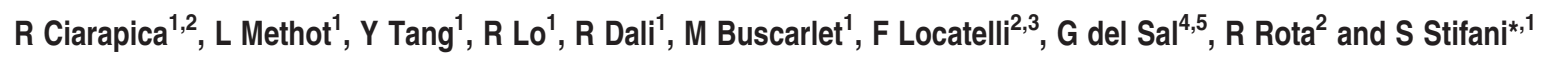

The Groucho/transducin-like Enhancer of split 1 (Gro/TLE1):Hes1 transcriptional repression complex acts in cerebral cortical neural progenitor cells to inhibit neuronal differentiation. The molecular mechanisms that regulate the anti-neurogenic function of the Gro/TLE1:Hes1 complex during cortical neurogenesis remain to be defined. Here we show that prolyl isomerase Pin1 (peptidyl-prolyl cis-trans isomerase NIMA-interacting 1) and homeodomain-interacting protein kinase 2 (HIPK2) are expressed in cortical neural progenitor cells and form a complex that interacts with the Gro/TLE1:Hes1 complex. This association depends on the enzymatic activities of both HIPK2 and Pin1, as well as on the association of Gro/TLE1 with Hes1, but is independent of the previously described Hes1-activated phosphorylation of Gro/TLE1. Interaction with the Pin1:HIPK2 complex results in Gro/TLE1 hyperphosphorylation and weakens both the transcriptional repression activity and the anti-neurogenic function of the Gro/ TLE1:Hes1 complex. These results provide evidence that HIPK2 and Pin1 work together to promote cortical neurogenesis, at least in part, by suppressing Gro/TLE1:Hes1-mediated inhibition of neuronal differentiation.

Cell Death and Differentiation (2014) 21, 321-332; doi:10.1038/cdd.2013.160; published online 22 November 2013

During mammalian brain development, the ventricular zone of the dorsolateral region of the alar telencephalon (neocortex) contains undifferentiated neural progenitor cells that initially undergo symmetric (proliferative) divisions to generate two new progenitor cells and later undergo asymmetric (differentiative) divisions to give rise to a new mitotic progenitor and a post-mitotic neuron. ${ }^{1,2}$ Precise regulation of the balance between the undifferentiated progenitor state and neuronal differentiation in the embryonic neocortex is essential for the correct development of the adult cerebral cortex, but the mechanisms regulating the progenitor-to-neuron transition in this brain region remain incompletely characterized.

Transcriptional repressors of the Hairy/Enhancer of split (Hes) family are important regulators of the balance between proliferation and differentiation during mouse cortical neurogenesis. Specifically, Hes1 acts downstream of Notch receptors to inhibit neuronal differentiation and promote maintenance of the undifferentiated state. ${ }^{3-5}$ In both invertebrate and vertebrate species, Notch-induced Hes proteins, such as Hes1, repress transcription together with the general transcriptional corepressors of the Groucho/transducin-like Enhancer of split (Gro/TLE) family. ${ }^{6-8}$ Gro/TLE1 is expressed in undifferentiated cortical neural progenitor cells in which Hes1 is also expressed ${ }^{9-11}$ and inhibits cortical neuronal differentiation in vivo and in vitro. ${ }^{10-12}$ Moreover, Hes1 and Gro/TLE1 physically interact, repress transcription together and localize to common promoter regions in vivo. ${ }^{13-15}$

Hes1 and Gro/TLE1 are expressed in neural progenitor cells during both the proliferative and differentiative phases of cortical neurogenesis, suggesting the existence of mechanisms that either promote or inhibit their anti-neurogenic function at different stages of cortical neuronal differentiation. Hes1 binds to the C-terminal WD40 repeat-containing domain of Gro/TLE1 through its own C-terminal tetrapeptide, WRPW. ${ }^{7,16}$ Complex formation with Hes1 results in Gro/ TLE1 recruitment to DNA, ${ }^{14,15}$ where Gro/TLE1 interacts with structural chromatin components. ${ }^{17}$ In addition, Hes1 binding causes increased Gro/TLE1 phosphorylation, a process previously termed 'cofactor-activated phosphorylation'. ${ }^{11,18}$ Inhibition of cofactor-activated phosphorylation by mutation of selected serine residues within the serine/proline-rich domain of Gro/TLE1 weakens the interaction of Gro/TLE1 with chromatin and impairs both the transcriptional repression ability and anti-neurogenic activity of this protein without affecting its interaction with Hes1. Thus, cofactor-activated phosphorylation of Gro/TLE1 induced by the binding of Hes1 has a positive role in the anti-neurogenic activity of the Gro/ TLE1:Hes1 complex. ${ }^{11,15}$

\footnotetext{
${ }^{1}$ Montreal Neurological Institute, McGill University, 3801 rue University, Montreal, Quebec H3A2B4, Canada; ${ }^{2}$ Department of Oncohematology, Ospedale Pediatrico Bambino Gesù, IRCCS, Roma, Italy; ${ }^{3}$ University of Pavia, Pavia, Italy; ${ }^{4}$ Laboratorio Nazionale CIB, Area Science Park, Trieste, Italy and ${ }^{5}$ Dipartimento di Scienze della Vita, Università degli Studi di Trieste, Trieste, Italy

${ }^{*}$ Corresponding author: S Stifani, Montreal Neurological Institute, McGill University, 3801 rue University, Montreal, Quebec H3A2B4, Canada. Tel: +1 514 3983946; Fax: +1 514 3981319; E-mail: stefano.stifani@mcgill.ca

Keywords: cerebral cortex; Groucho/transducin-like Enhancer of split; Hes1; HIPK2; neuronal differentiation; Pin1

Abbreviations: ChIP, chromatin immunoprecipitation; GFP, green fluorescent protein; Gro, Groucho; Hes, Hairy/Enhancer of split; HIPK2, homeodomain-interacting protein kinase 2; Pin1, peptidyl-prolyl cis-trans isomerase NIMA-interacting 1; q-PCR, quantitative PCR; TLE, transducin-like Enhancer of split

Received 24.3.13; revised 10.9.13; accepted 9.10.13; Edited by D Kaplan; published online 22.11.2013
} 
In this study, we sought to characterize additional molecular mechanisms underlying the regulation of Gro/TLE1:Hes1 function during cortical neuronal differentiation. Here we provide evidence that the phosphorylation-dependent prolyl isomerase Pin1 (peptidyl-prolyl cis-trans isomerase NIMAinteracting 1), which recognizes phosphorylated Ser/Thr-Pro sites and catalyzes proline cis/trans isomerization, ${ }^{19,20}$ and the protein kinase homeodomain-interacting protein kinase 2 $(\text { HIPK2) })^{21,22}$ are expressed during cortical neurogenesis and form a complex. The Pin1:HIPK2 complex interacts with Gro/TLE1:Hes1 and inhibits the anti-neurogenic function of these proteins during cortical neuronal differentiation. These findings reveal a previously uncharacterized role for a complex of Pin1 and HIPK2 in the promotion of cortical neurogenesis at least in part by antagonizing Gro/TLE1:Hes1mediated inhibition of neuronal differentiation.

\section{Results}

Pin1 interacts with Gro/TLE1 in the developing cerebral cortex and is important for cortical neuronal differentiation. Gro/TLE1 undergoes increased phosphorylation during neurogenesis ${ }^{18,23}$ and contains several putative Ser-Pro and Thr-Pro phosphorylation sites. At least one of these sites, ${ }^{286}$ Ser-Pro, is phosphorylated in vivo, ${ }^{15}$ suggesting that Gro/TLE1 might be a substrate for the ubiquitous prolyl isomerase Pin1. This enzyme catalyzes proline cis/trans isomerization at phosphorylated Ser/Thr-Pro sites, a process that often results in conformational changes that modulate the activity of the Pin1 protein substrates. ${ }^{19,20}$ We observed that in mouse embryos at embryonic day (E) 13.5, a period of active cortical neuronal differentiation, endogenous Pin1 was coexpressed with endogenous Gro/ TLE proteins in both the ventricular zone of the cerebral cortex, where undifferentiated neural progenitor cells are located, and in the developing cortical plate, where migrated post-mitotic neurons come to reside (Figure 1a). Overlapping expression of Pin1 and Gro/TLE was also observed in primary cultures of cortical neural progenitor cells established from E13.5 mouse embryos (Supplementary Figure S1a). More importantly, immunoprecipitation of endogenous Gro/TLE1 from mouse cerebral cortices dissected at E13.5 resulted in the coprecipitation of endogenous Pin1 (Figure 1b). Together, these results show that Pin1 and Gro/TLE1 are coexpressed and interact during cerebral cortex neurogenesis.

On the basis of these observations, we examined whether the association of Gro/TLE1 with Pin1 might be modulated by Hes1, a key mediator of Gro/TLE1 anti-neurogenic activity. ${ }^{11,12,14,18}$ HEK293 cells, which express endogenous Gro/TLE1 and Hes 1, ${ }^{15,18}$ were transfected with Pin1 and Gro/ TLE1 in the absence or presence of exogenous Hes1, followed by immunoprecipitation with anti-Gro/TLE1 antibody. Both Hes1 and Pin1 coprecipitated with Gro/TLE1, and Pin1 associated more efficiently with Gro/TLE1 in the presence of increased levels of Hes1 (Figure 1c). These observations provide evidence that Hes 1 is important for the association of Pin1 with Gro/TLE1.

To begin to investigate the involvement of Pin1 in the antineurogenic function of the Gro/TLE1:Hes1 complex, we determined the effect of Pin1 inhibition on cortical neuronal differentiation in vitro. Pharmacological inhibition of endogenous Pin1 in primary cultures of cortical progenitor cells using the reagent juglone ${ }^{24}$ caused a reduced generation of cells expressing the neuronal markers MAP2 and type III $\beta$-tubulin and a parallel accumulation of cells expressing the proliferation marker Ki67 and the undifferentiated neural progenitor marker nestin, compared with control conditions (Figures 1d and e). Consistent with these results, exposure of cortical progenitor cells to juglone caused both increased levels of phosphorylated histone $\mathrm{H} 3$, a marker of mitotic cells, and decreased amounts of type III $\beta$-tubulin and GAP-43, another protein expressed in differentiated neurons (Figure 1f). The same effects were observed after silencing of Pin1 in cortical progenitor cells using lentiviral-mediated expression of a Pin1 short hairpin (sh) RNA reagent, but not when a control non-silencing shRNA was used (Figure 1g). Taken together with the demonstration that knockout of mouse Pin1 causes decreased neuronal differentiation in cultured cortical progenitor cells, ${ }^{25}$ these results show that Pin1 promotes cortical neurogenesis. Moreover, they provide evidence that Gro/TLE1:Hes1 and Pin1 have opposite biological functions during cortical neuronal development.

Pin1 interacts with Gro/TLE1:Hes1 complex together with HIPK2. To characterize the mechanisms underlying Pin1 interaction with Gro/TLE1, we focused on the protein kinase HIPK2, which commonly phosphorylates Ser/Thr-Pro sites $^{21}$ and was implicated in the phosphorylation of Gro/TLE in Drosophila. ${ }^{26,27}$ Endogenous Gro/TLE1, Pin1 and HIPK2 were expressed in dissected cerebral cortices from E13.5 mouse embryos (Supplementary Figure S1b). Furthermore, HIPK2 was coexpressed with both Gro/TLE1 and Pin1 in primary cultures of cortical neural progenitor cells (Supplementary Figures S1c and d). More importantly, immunoprecipitation of Pin1 from HEK293 cells transfected with Gro/TLE1, Hes1 and HIPK2 resulted not only in the coprecipitation of the former three proteins but also in the specific coprecipitation of HIPK2 (Figure 2a, lane 5; and Supplementary Figure S2). The coprecipitation of Gro/TLE1 and Hes1 with Pin1 was significantly weaker, or even undetectable, in the absence of exogenous HIPK2 or in the presence of a catalytically inactive form of HIPK2, termed HIPK2(K221R) ${ }^{28}$ (Figure 2a; see lanes 4-6). As previously shown, the catalytically inactive form of HIPK2 migrated faster than wild-type HIPK2. ${ }^{26}$ Moreover, neither Gro/TLE1 nor Hes1 coprecipitated with Pin1 and HIPK2 when Hes1 was mutated to prevent Gro/TLE1 binding by deleting the C-terminal WRPW sequence required for binding to Gro/TLE $\left(\right.$ Hes1 1 WRPW) ${ }^{13,15,29,30}$ (Figure 2a; see lanes 5 and 7), suggesting further that Pin1 interacts with the Gro/TLE1:Hes1 complex, rather than with Gro/TLE1 or Hes1 alone.

These studies also revealed that both HIPK2 and HIPK2(K221R) readily coprecipitated with Pin1 irrespective of the coprecipitation of Gro/TLE1 and Hes1, showing that HIPK2 and Pin1 form a complex (Figure 2a, see lanes 5-7). The Pin1:HIPK2 interaction was dependent on the prolyl isomerase enzymatic activity of Pin1, because immunoprecipitation of a catalytically inactive Pin1 mutant, termed Pin1(S67E), ${ }^{31}$ did not result in the coprecipitation of HIPK2, 
nor that of Gro/TLE or Hes1 (Figure 2b, see lanes 2 and 3). Together, these results show that Pin1 interacts with HIPK2 and that this complex associates with Gro/TLE1:Hes1 in a manner that depends on both the enzymatic activity of Pin1 and the catalytic activity of HIPK2.

HIPK2 mediates Gro/TLE1 phosphorylation in Hes1dependent manner. We next investigated whether the association of the Pin1:HIPK2 complex with Gro/TLE1:Hes1 was correlated with changes in Gro/TLE1 phosphorylation. Binding of Gro/TLE1 to Hes1, as well as other transcription factors such as Runx1, results in 'cofactor-activated phosphorylation' of Gro/TLE1, a process that can be detected through a characteristic gel mobility shift ${ }^{12,15,18}$ (Figure 3a; see lanes 1 and 2; 4 and 5). The shift in Gro/TLE1 mobility induced by either Hes1 or Runx1 was enhanced by the coexpression of HIPK2 (Figure 3a; see lanes 2 and 3; 5 and 6). The HIPK2-mediated increase of more slowly migrating Gro/TLE1 was abolished in the presence of calf intestinal phosphatase, showing that it was the result of hyperphosphorylation (Figure 3b; see lanes 3 and 6). Consistent with this result, we observed no effect on Gro/TLE1 gel mobility when the catalytically inactive HIPK2(K221R) was used (Figure 3c; see lanes 2-4; Figure 3d(i), see lanes 2-4). Importantly, the expression of HIPK2 had no detectable consequence on Gro/TLE1 gel mobility in the absence of either Hes1 or Runx1 (Figure 3c, see lanes 3 and 5; Figure 3d(i), see lanes 3 and 5).

On the basis of these results, we determined whether the requirement of Hes1 binding for HIPK2-mediated phosphorylation of Gro/TLE1 reflected a need for either prior complex formation between Hes1 and Gro/TLE1 or prior Hes1induced cofactor-activated phosphorylation of the latter. To distinguish between these possibilities, we uncoupled these two events by taking advantage of previously described Gro/ TLE1 proteins harboring point mutations that reduce/block cofactor-activated phosphorylation without interfering with the binding of Gro/TLE1 to Hes1. Two separate proteins, Gro/TLE1(S239A) and Gro/TLE1(S298A), previously shown to display a markedly decreased cofactor-activated phosphorylation $^{11,15}$ (Figure $3 d(i-i i i)$, see lanes 1 and 2), underwent a significant electrophoretic mobility shift in the presence of HIPK2, suggesting that they were still competent to undergo HIPK2-mediated phosphorylation (Figure 3d(ii and iii), lane 3). Two other mutated Gro/TLE proteins, Gro/TLE1(S286A) and Gro/TLE1(S289A), carrying point mutations that completely block cofactor-activated phosphorylation, ${ }^{15}$ exhibited a detectable gel retardation in the presence of HIPK2 but not HIPK2(K221R) (Figure 3d(iv and v), lanes 3 and 4). This mobility shift was also observed when Ser-286 was mutated to Glu (S286E), a mutation that also completely blocks Gro/TLE1 cofactor-activated phosphorylation but not Hes1 binding ${ }^{15}$ (Figure $3 e$, see lanes 4-6). Both Gro/TLE1(S286A) and Gro/TLE1(S286E) coprecipitated with Pin1, HIPK2 and Hes1 similar to wild-type Gro/TLE1 (Figure 3e, see lanes 3, 5 and 6). Together, these findings show that HIPK2 mediates Gro/TLE1 hyperphosphorylation in a Hes1-dependent manner. This requirement is not due to a necessity for prior Hes1-induced cofactor-activated phosphorylation of Gro/TLE1 and likely underscores a need for Hes1 binding-mediated conformational changes in Gro/TLE1.

Pin1 and HIPK2 weaken the transcriptional repressor activity of Gro/TLE1:Hes1. We next determined the effect of HIPK2-mediated Gro/TLE1 hyperphosphorylation on the transcriptional repressor function the Gro/TLE1:Hes1 complex. We observed that the previously demonstrated ${ }^{11-13}$ ability of Hes1 to repress transcription in cells in which Gro/TLE proteins are endogenously expressed was blocked by a dominant-negative form of Gro/TLE, termed Gro/TLE1 $\Delta \mathrm{Q},{ }^{32}$ showing that Hes1-mediated transcriptional repression depends on Gro/TLE corepressor activity (Figure 4a, see bars 2 and 3). The coexpression of Pin1 and HIPK2 also inhibited Hes1-mediated transcriptional repression, albeit not to the same extent as Gro/TLE-dominant inhibition (Figure 4a, see bars 2 and 4), possibly suggestive of preexisting endogenous Pin1:HIPK2-mediated inhibition. In agreement with the robust endogenous expression of Pin1 in virtually all cell types, ${ }^{19,20}$ expression of HIPK2 alone was sufficient to antagonize both Hes1-mediated transcriptional repression and the ability of exogenous Gro/TLE1 to enhance the latter effect (Figure $4 \mathrm{~b}$, see bars $2-4$ and 6 ). These assays were performed under experimental conditions where an initially suboptimal transcriptional repression by Hes 1 was potentiated by coexpression of Gro/TLE1. ${ }^{12,15}$ These results were correlated with a marked shift of Gro/TLE1 to a slower electrophoretic mobility in the presence of exogenous HIPK2 (Figure 4c, see lanes 3 and 4). HIPK2(K221R) did not elicit either of these effects (Figures $4 \mathrm{~b}$ and $\mathrm{c}$, lane 5). These findings provide evidence that HIPK2-mediated hyperphosphorylation antagonizes the transcriptional repression function of Gro/TLE1:Hes1.

To determine the mechanisms underlying this inhibitory effect, we examined whether HIPK2 might weaken the association of Gro/TLE1 with Hes1 (Gro/TLE binding-incompetent Hes1 1 WRPW was used as a negative control). Coimmunoprecipitation experiments showed that neither HIPK2 nor HIPK2(K221R) had a detectable effect on the formation of the Gro/TLE1:Hes1 complex (Figure 4d, see lanes 4-6). As shown previously, ${ }^{18} \mathrm{Gro} / \mathrm{TLE} 1$ was recovered more robustly in the cell lysates when Hes1 1 WRPW was used, due to the lack of Hes1-mediated DNA recruitment and association with chromatin (Figure 4d, see lanes 2 and 3). These results suggest that HIPK2-mediated phosphorylation does not interfere with the association of Gro/TLE1 with Hes 1.

We therefore examined whether HIPK2 might exert a negative effect on the association of Gro/TLE1:Hes1 with chromatin. Using a biochemical fractionation approach previously adopted to separate the chromatin-bound Gro/TLE1 population from the chromatin-unbound portion, ${ }^{12,18,23}$ we observed that Gro/TLE1 was recovered mainly in the nonchromatin ('post-nuclear supernatant') fraction in the absence of Hes1, whereas a significant amount of Gro/TLE1 was found in the chromatin fraction together with Hes1 (Figure 5a, see lanes 1 and 2). The coexpression of HIPK2 caused a significant decrease in the amount of Gro/TLE1 recovered in the chromatin fraction in the presence of Hes1, with a concomitant increase in the post-nuclear supernatant (Figure 5a, see lanes 3 and 4; Figure 5b, see lanes 2-5). 
a
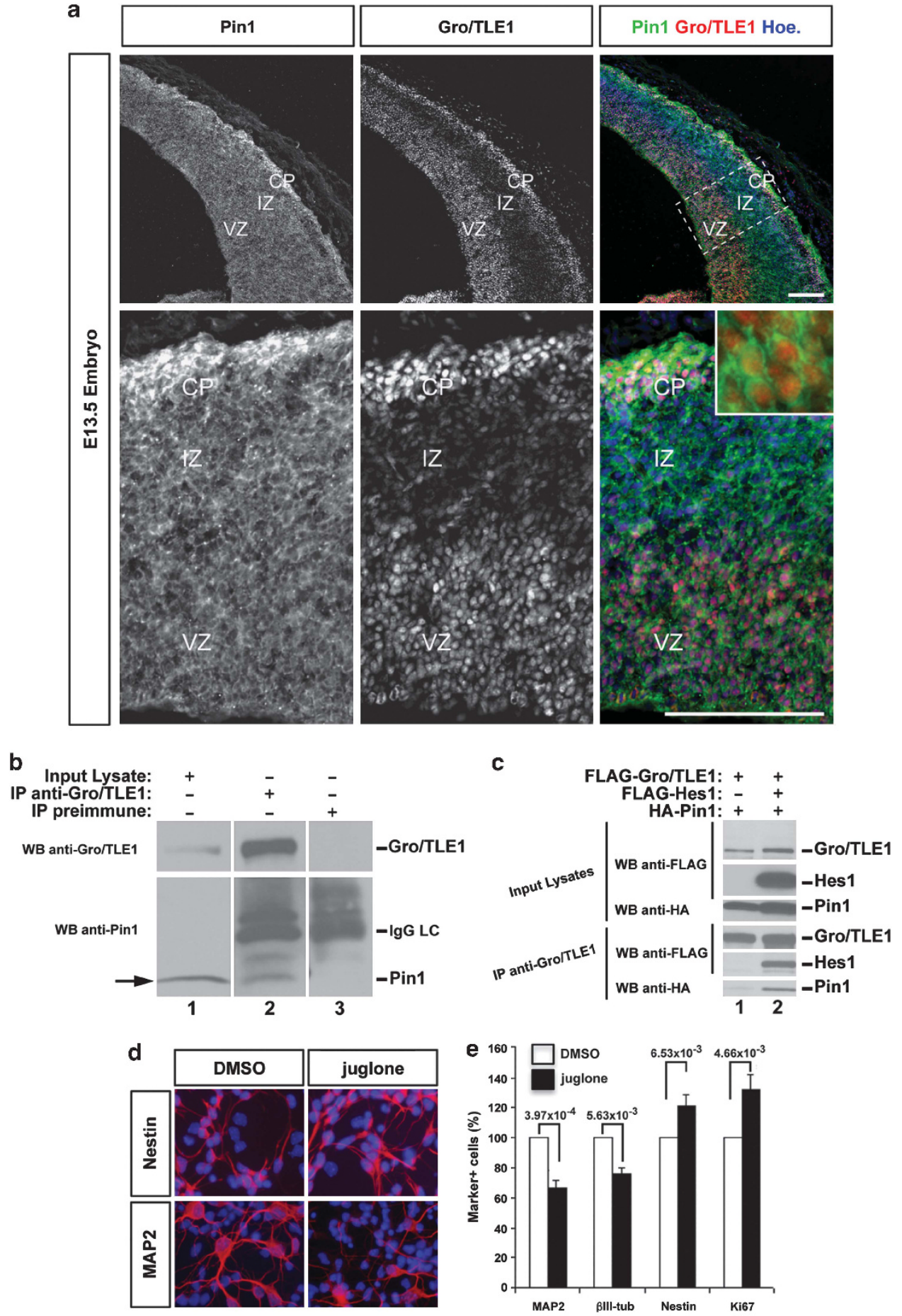

f

g

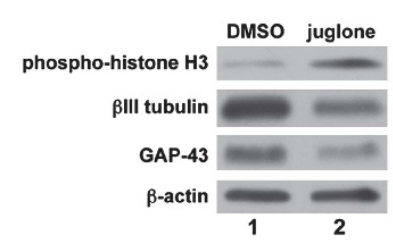
$\begin{gathered}\text { Non-silen. } \\ \text { shRNA }\end{gathered}$ Pin1
shRNA

phospho-histone H3

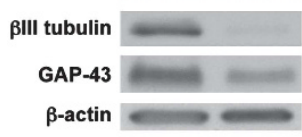

12 

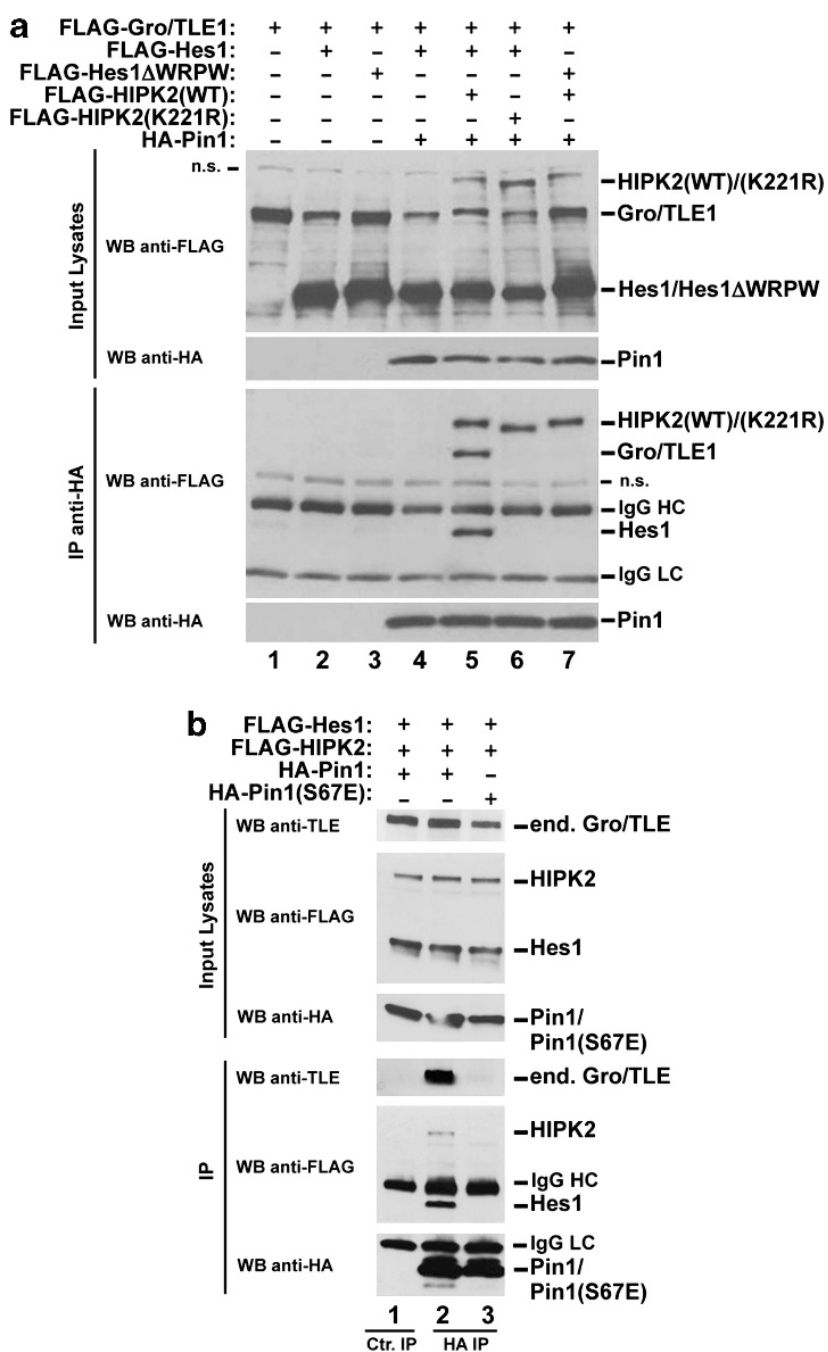

Figure 2 Interaction of Gro/TLE1:Hes1 complex with Pin1 and HIPK2. Coimmunoprecipitation studies using HEK293 cells transfected with the indicated combinations of proteins. (a) Immunoprecipitation (IP) of HA-tagged Pin1 using antiHA antibody results in the coprecipitation of both HIPK2(WT) and HIPK2(K221R), as revealed by western blot (WB) analysis. Gro/TLE1 and Hes1 coprecipitate efficiently with Pin1 and HIPK2, but not when HIPK2(K221R) is used, nor in the presence of Hes1 ( $\triangle W R P W)$. IgG HC and IgG LC, immunoglobulin $G$ heavy or light chain, respectively; 'n.s.', non-specific band. (b) Immunoprecipitation of HA-tagged Pin1(S67E) using anti-HA antibody (Pin1-HA IP) does not result in detectable coprecipitation of HIPK2(WT), Gro/TLE1 or Hes1, in contrast to wild-type Pin1. Control immunoprecipitation (Ctr. IP) with preimmune serum is shown in lane 1
HIPK2(K221R) did not have the same effect (Figure 5b, lane 6). HIPK2(K221R) appeared to have a stronger association with the chromatin fraction than HIPK2(WT) (Figure 5b, see lanes 5 and 6). Similar to Gro/TLE1, the amount of Hes1 recovered in the chromatin fraction was also reduced in the presence of HIPK2, and Hes1 appeared to migrate more slowly (Figure 5b, see lanes 2-5). For unknown reasons, Hes1 expression seemed to be negatively affected by the expression of HIPK2(K221R) (Figure 5b, lane 6; see also Figure 4c, lane 7).

In agreement with the previous findings, chromatin immunoprecipitation (ChIP) experiments showed that binding of exogenous FLAG-tagged Hes1 to the promoter of the ASCL1 gene, a previously described transcriptional target of Hes $1,{ }^{14}$ was significantly reduced in the presence of exogenous Pin1 and HIPK2 (Figure 5c, see lanes 2 and 6; Figure 5d). Moreover, electrophoretic mobility shift assays showed that the mobility of a double-stranded oligonucleotide containing two Hes1 binding sites was retarded in the presence of exogenous Hes 1 and that this mobility shift was abolished by the coexpression of Pin1 and HIPK2 (Figure 5e, see lanes 4 and 6). The exogenous expression of HIPK2 alone had a weaker effect, suggesting that the observed outcome was mediated by a Pin1:HIPK2 complex (Figure 5e, see lanes 4-6). Taken together, these findings show that Pin1 and HIPK2 act as negative regulators of the transcriptional repressor activity of the Gro/TLE1:Hes1 complex, at least in part by weakening the association of the latter with chromatin.

Pin1 and HIPK2 inhibit the anti-neurogenic activity of Gro/TLE1:Hes1. On the basis of the previous results, we examined whether Pin1 and HIPK2 would antagonize the anti-neurogenic activity of Gro/TLE1:Hes1. This possibility was first examined in SH-SY5Y neuroblastoma cells, which can be induced to undergo neuronal differentiation in vitro upon treatment with retinoic acid. ${ }^{33,34} \mathrm{SH}-\mathrm{SY} 5 \mathrm{Y}$ cells do not normally express the protein Tau, a marker of differentiated neurons, but expression of this protein is induced by treatment with retinoic acid ${ }^{34,35}$ (Figure 6a, left-hand panel). We first compared the numbers of retinoic acid-treated SH-SY5Y cells expressing either Ki67 or Tau after transfection of plasmids expressing green fluorescent protein (GFP) alone (control) or together with Gro/TLE1 and Hes1. Exogenous Gro/TLE1:Hes1 caused a reduction in the number of Tau-positive SH-SY5Y cells and a parallel

Figure 1 Interaction of Gro/TLE1 with Pin1. (a) Double-label immunofluorescence analysis of Gro/TLE and Pin1 protein expression in the developing mouse cerebral cortex. Endogenous Pin1 and Gro/TLE proteins (detected using a panGro/TLE antibody) are coexpressed in the ventricular zone (VZ) and cortical plate (CP) of E13.5 cerebral cortex. Gro/TLE, but not Pin1, expression is transiently downregulated in the intermediate zone (IZ), where newly born neurons are located. Bottom row contains highmagnification views of the boxed area in top row. Inset in right-hand panel in bottom row depicts examples of double-labeled cells. Counterstaining of cell nuclei with Hoechst is shown. Scale bar: $100 \mu \mathrm{m}$. (b and c) Coimmunoprecipitation studies. (b) Lysates from dissected E13.5 mouse cerebral cortices were subjected to immunoprecipitation (IP) of endogenous Gro/TLE1, followed by western blot (WB) analysis of the expression of Gro/TLE1 and Pin1. In this and succeeding immunoprecipitation experiments immunoprecipitates are shown together with $1 / 10$ of each input lysate. Arrow points to the position of Pin1 immunoreactive band. IgG LC, immunoglobulin G light chain. To avoid possible spill over artifacts, gel electrophoresis was performed leaving empty lanes between samples. (c) Lysates from transfected HEK293 cells were subjected to immunoprecipitation of FLAG-tagged Gro/TLE1 using anti-Gro/TLE1 antibody followed by western blot analysis of the expression of FLAG-tagged and HA-tagged proteins, as indicated. (d-g) Effect of Pin1 inhibition on cortical neuronal differentiation in vitro. (d and e) Primary cultures of E13.5 mouse cortical progenitor cells were treated with either the Pin1 pharmacological inhibitor juglone or DMSO, as control, followed by comparison of the numbers of cells (nuclei visualized through Hoechst counterstaining) that expressed either the neuronal markers MAP2 and type III $\beta$-tubulin or the progenitor markers nestin and Ki67. Data are shown as mean \pm S.D. of four separate experiments performed in duplicates; $P$-values, two-tailed Student $t$-test. (f) Lysates from control or juglone-treated cortical progenitor cells were subjected to western blot with antibodies against the mitotic cell marker phospho-histone $\mathrm{H} 3$ or the neuronal markers type III $\beta$-tubulin and GAP-43. Expression of $\beta$-actin is shown as gel loading control. (g) Lysates from cortical progenitor cells transduced with either non-silencing shRNA or Pin1 shRNA reagents were subjected to western blot with antibodies against the indicated proteins 
a

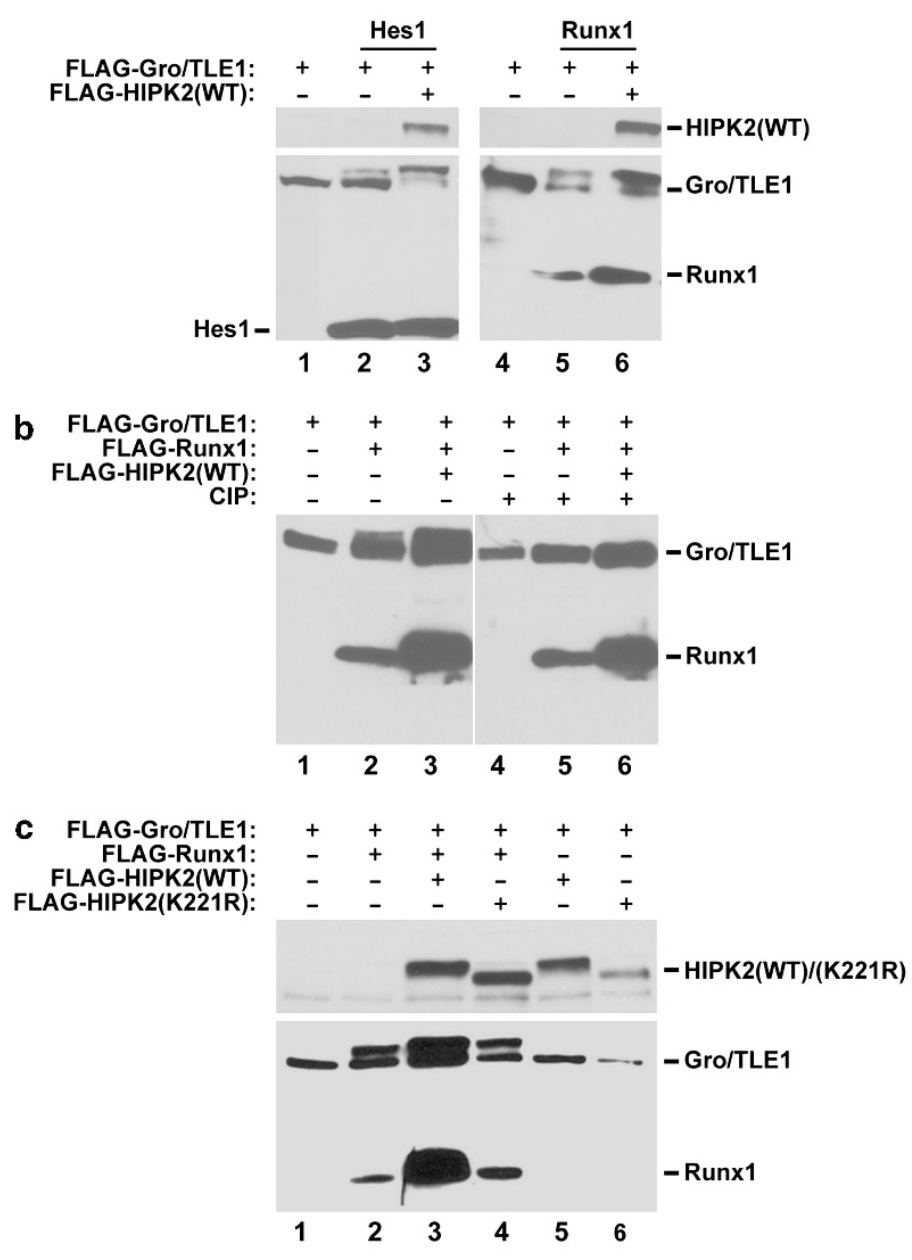

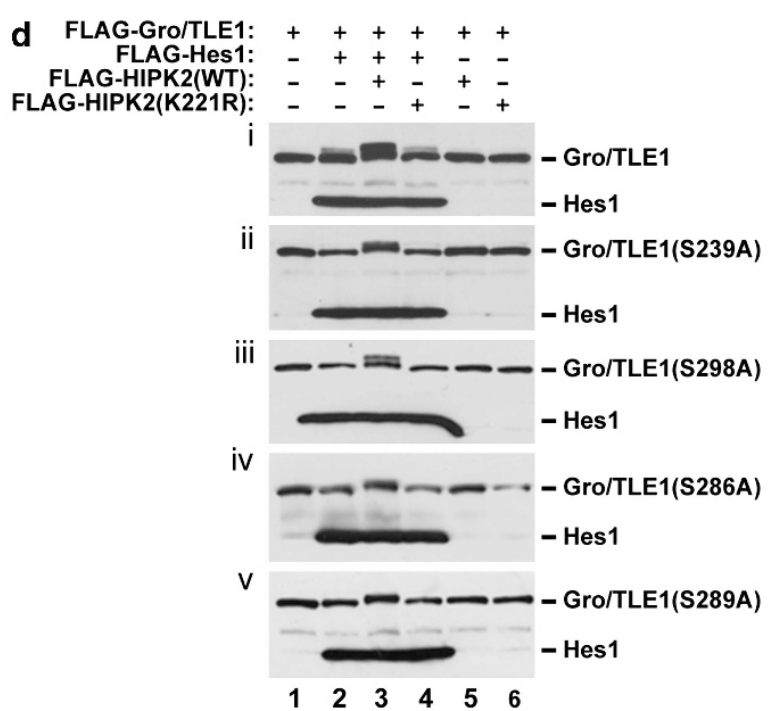

e

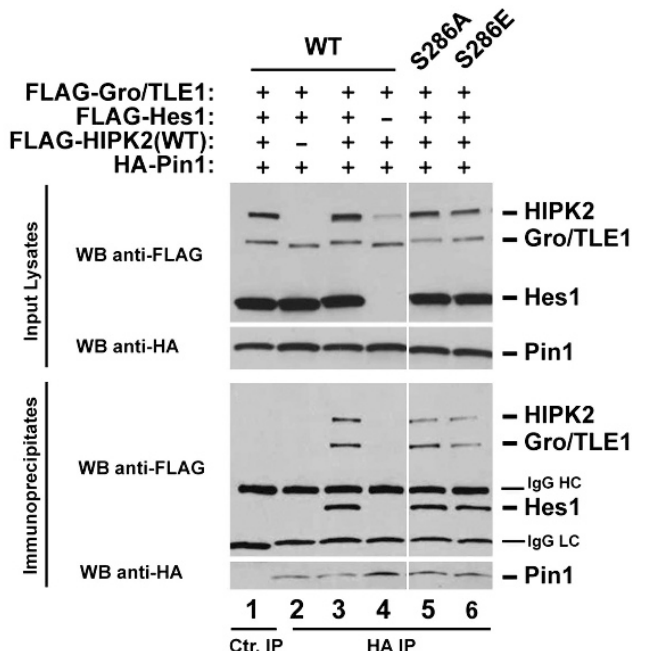

Figure 3 HIPK2-mediated Gro/TLE1 hyperphosphorylation is independent of cofactor-activated phosphorylation. (a-c) Western blot analysis of Gro/TLE1 in the presence of its DNA binding partners Hes1 or Runx1, as indicated, alone or together with HIPK2(WT) or HIPK2(K221R) using anti-FLAG antibody. All of these proteins contained a FLAG epitope. (a) Both Hes1 and Runx1 cause a shift in Gro/TLE1 electrophoretic mobility and this gel retardation is enhanced by coexpression of HIPK2. (b) The retardation of Gro/TLE1 gel mobility observed in the presence of Runx1 alone or the combination of Runx1 and HIPK2 is abolished by incubation of cell lysates with calf intestinal phosphatase (CIP) before electrophoresis. The presence of HIPK2 leads to increased levels of Runx1 and Gro/TLE1. (c) HIPK2(WT), but not HIPK2(K221R), causes retarded mobility and increased expression of Gro/TLE1 only in the presence of Runx1. (d) Western blot analysis of Gro/TLE1 or the indicated Gro/TLE1 point mutated forms in the presence of Hes1 alone or together with HIPK2(WT) or HIPK2(K221R) using anti-FLAG antibody. (i) HIPK2(WT), but not HIPK2(K221R), causes retarded mobility of Gro/TLE1 only in the presence of Hes1. (ii-v) Cofactor-activated phosphorylation-impaired Gro/TLE1 mutants undergo a shift to a slower mobility in the presence of HIPK2(WT) but not HIPK2(K221R). (e) Coimmunoprecipitation studies using transfected HEK293 cells. Cofactor-activated phosphorylation-impaired forms of Gro/TLE1-bearing mutations S286A or S286E form complexes with Pin1, HIPK2 and Hes1 similar to wild-type (WT) Gro/TLE1, as revealed by western blot (WB) analysis after immunoprecipitation of HA-Pin1 with anti-HA antibody. Control immunoprecipitation (IP) with preimmune serum is shown in lane 1. IgG HC and IgG LC, immunoglobulin G heavy or light chain, respectively

increase in Ki67-positive cells (Figure 6a, right-hand panel, see bars 1-4). More importantly, these effects were antagonized by the coexpression of Pin1 and HIPK2 but not Pin1 and HIPK2(K221R) (Figure 6a, right-hand panel, see bars 3-8). Expression of HIPK2 alone did not cause a significant increase in neuronal differentiation, but we observed a trend toward increased numbers of Ki67-positive cells in the absence of exogenous Gro/TLE1:Hes1 (Figure 6a, right-hand panel, see bars 1, 2 and 9, 10).

To confirm these observations, we examined the effect of Pin1 and HIPK2 on the anti-neurogenic activity of Gro/TLE1 and Hes1 in primary cultures of cortical progenitor cells.
Compared with the expression of GFP alone, coexpression of Gro/TLE1 and Hes1 resulted in a significant increase in the number of $\mathrm{GFP}^{+}$cells expressing the undifferentiated neural progenitor markers nestin and Sox2 (Figures $6 \mathrm{~b}$ and $c$, see bars 1 and 2 in right-hand panels), and the mitotic marker Ki67 (Supplementary Figure S4), with a concomitant decrease in the number of cells expressing the neuronal markers MAP2 and NeuN (Figures 6d and e, see bars 1 and 2 in right-hand panels), as well as type III $\beta$-tubulin (Supplementary Figure S4). More importantly, these effects were blocked by the coexpression of Pin1 and HIPK2 but not by the coexpression of Pin1 and HIPK2(K221R) (Figures 6b-e, see bars 2-4 in 
a

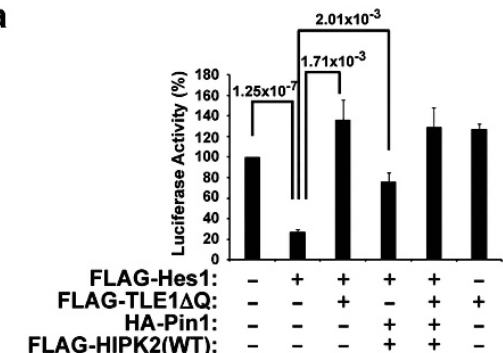

b

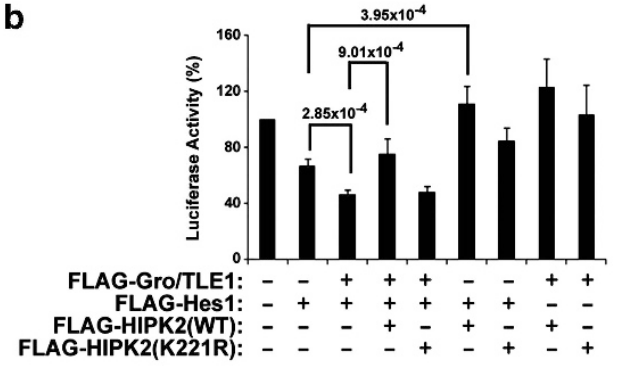

C
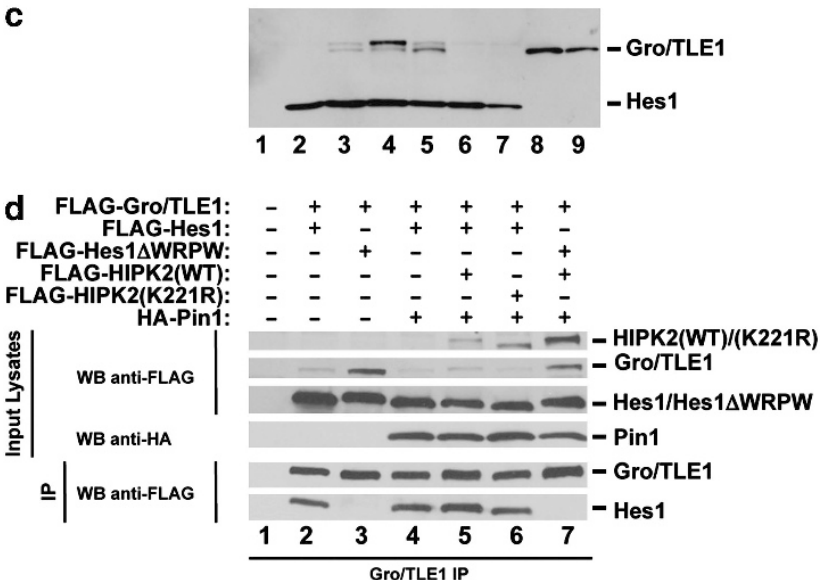

Figure 4 HIPK2-mediated phosphorylation of Gro/TLE1 is correlated with decreased transcriptional corepressor activity. (a-c) Analysis of the transcriptional corepressor activity of Gro/TLE1 for Hes1 in transient transfection/transcription assays. HEK293 cells were transfected with a reporter construct harboring the luciferase gene under the control of the neurogenin3 promoter linked to Hes 1 binding sites alone or in combination with plasmids driving expression of the indicated proteins. Luciferase activity in the absence of any cotransfected plasmid was considered as $100 \%$. (a) Hes1 represses transcription of the reporter gene and this effect depends on endogenous Gro/TLE because it can be blocked by Gro/ $T L E 1 \Delta Q$, a validated dominant-negative form of Gro/TLE. Transcriptional repression mediated by Gro/TLE:Hes1 is significantly decreased by coexpression of Pin1 and HIPK2 (mean \pm S.D. of four separate experiments performed in duplicates; $P$-values, two-tailed Student $t$-test). (b) Exogenous Gro/TLE1 potentiates Hes1-mediated transcriptional repression when the latter is tested under suboptimal conditions. Expression of HIPK2(WT), but not HIPK2(K221R), antagonizes Hes1-mediated transcriptional repression both in the absence and presence of exogenous Gro/TLE1 (mean \pm S.D. of four separate experiments performed in duplicates; $P$-values, two-tailed Student $t$-test). (c) Western blot analysis of Gro/TLE1 and Hes1 used in the transcription assays depicted in panel (b). (d) Coimmunoprecipitation studies using HEK293 cells transfected with the indicated combinations of proteins, followed by immunoprecipitation (IP) using anti-Gro/TLE1 antibody. Hes1, but not Hes1 $\triangle$ WRPW, coprecipitates with Gro/TLE1 in the absence and presence of Pin1 alone or in combination with HIPK2, as determined by western blot (WB) analysis right-hand panels; Supplementary Figure S4). Coexpression of either Pin1 alone or HIPK2 alone showed a trend toward a reduced anti-neurogenic effect by Gro/TLE1 and Hes1, but these results were not as pronounced as when Pin1 and HIPK2 were expressed together (Figures $6 \mathrm{~b}-\mathrm{e}$, bars 5 and 6 in right-hand panels; Supplementary Figure S4). Together, these results provide evidence that Pin1 and HIPK2 can antagonize the anti-neurogenic activity of the Gro/TLE:Hes1 complex in cortical neural progenitor cells.

\section{Discussion}

The anti-neurogenic activity of Gro/TLE1 depends on two sequential events, basal phosphorylation and cofactor (e.g., Hes1)-activated phosphorylation. Together, these processes establish a post-translational modification state that mediates strong association of Gro/TLE1-containing complexes with chromatin and optimal transcriptional repression function ${ }^{11,15,18}$ (Figure 7). In this study, we have obtained evidence that Hes 1 binding not only triggers the cofactoractivated phosphorylation of Gro/TLE1 but also promotes the association of the Gro/TLE1:Hes1 complex with Pin1 and HIPK2 (Figure 7). This higher-order association depends on the formation of a complex of Gro/TLE1 and Hes1 and also on the enzymatic activity of Pin1 (required for the Pin1:HIPK2 interaction) and the catalytic activity of HIPK2 (needed for Pin1:HIPK2 association with Gro/TLE1:Hes1).

The recruitment of Pin1 and HIPK2 to the Gro/TLE1:Hes1 complex leads to Gro/TLE1 hyperphosphorylation. This effect requires the catalytic activity of HIPK2 and does not occur in the absence of Hes1. The latter observation raised the possibility that the Pin1:HIPK2 complex might be recruited to Gro/TLE1 through Pin1-mediated binding to Ser/ThrPro site(s) generated by Hes1-mediated cofactor-activated phosphorylation. However, our studies have shown that cofactor-activated phosphorylation in response to Hes1 binding is not required for the association of Gro/TLE1:Hes1 with Pin1 and HIPK2, suggesting instead that Hes1 binding induces conformational changes in Gro/TLE1 that expose previously unavailable Ser/Thr-Pro site(s) that become phosphorylated by HIPK2. It is possible that Hes1 might also be a substrate of HIPK2-mediated phosphorylation because Hes1 electrophoretic mobility was reduced in the presence of HIPK2, but not the catalytically inactive form of HIPK2. However, this possibility remains to be determined.

Our results suggest that HIPK2-mediated phosphorylation of Gro/TLE1 has the potential to generate phosho-Ser/ThrPro sites that may become targets of the enzymatic activity of Pin1 bound to HIPK2 (Figure 7). Although the possibility that Gro/TLE1 (and possibly Hes1) might be a substrate of Pin1 prolyl isomerase activity remains to be tested, it is reasonable to speculate that Pin 1 could then mediate prolyl isomerization events at those specific sites, leading to conformational changes that could in turn modulate the biochemical activity of Gro/TLE1:Hes1.

We have also provided evidence that Pin1 and HIPK2 impair transcriptional repression mediated by Gro/ TLE1:Hes1. This effect, likely the result of changes in phosphorylation or/and isomerization at specific Ser/Thr-Pro sites, is caused at least in part by the weakening of the binding 


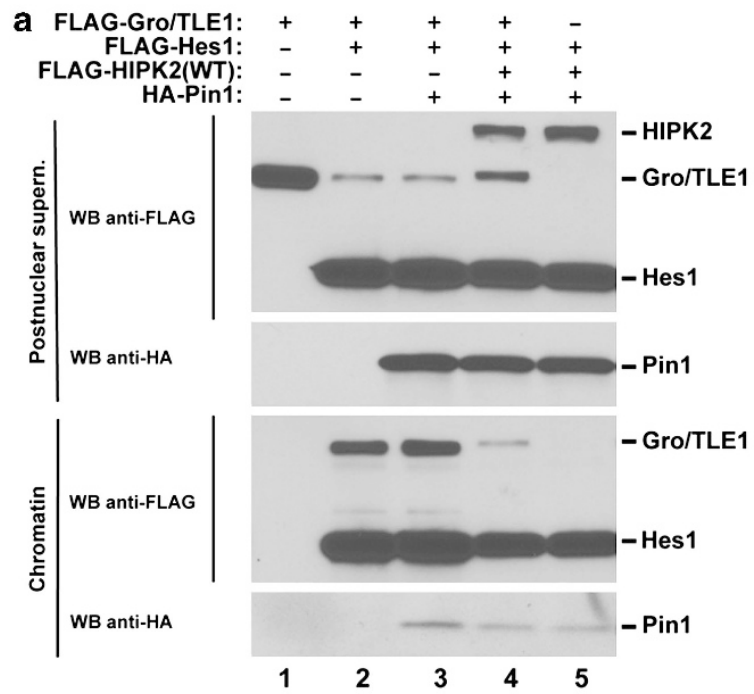

\section{b}

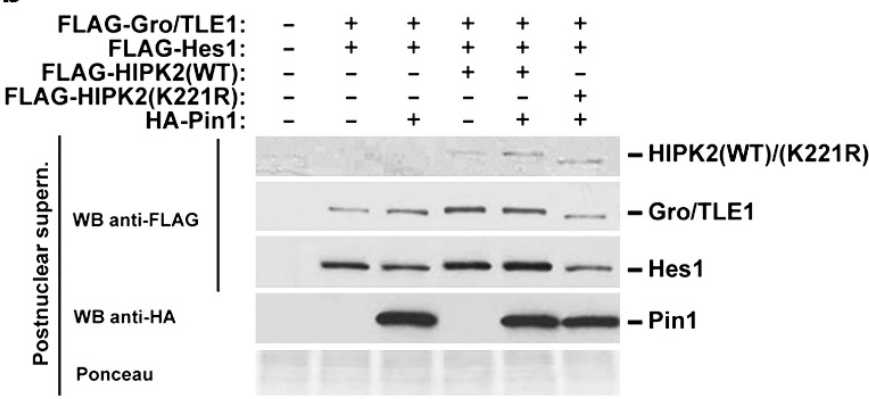

d

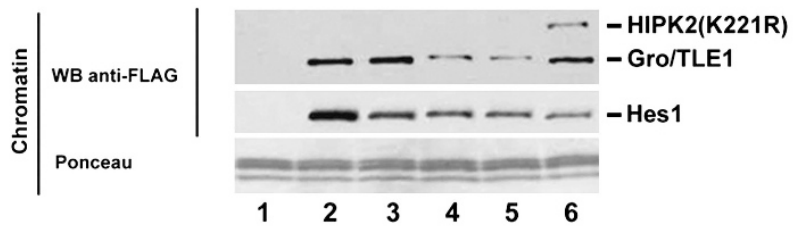

$1.57 \times 10^{-2}$

e
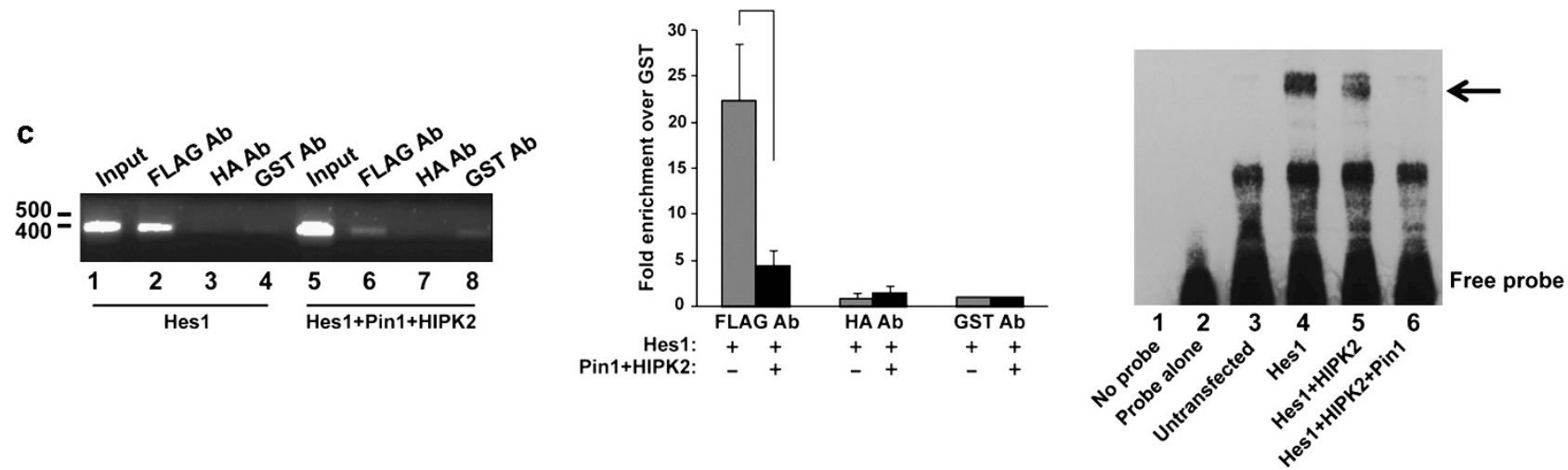

Figure 5 HIPK2 weakens the chromatin association of the Gro/TLE1:Hes1 complex. (a and $\mathbf{b}$ ) western blot (WB) analysis of Gro/TLE1 and Hes1 distribution in postnuclear supernatant and chromatin fractions from HEK293 cells transfected with the indicated combinations of proteins. (a) The nuclear retention of Gro/TLE1 is increased in the presence of Hes1 and this effect is antagonized by HIPK2 and Pin1 but not by Pin1 alone. A fraction of Pin1 is recovered in the chromatin fraction. (b) HIPK2 causes weakened association of both Gro/TLE1 and Hes1 with chromatin and this effect is correlated with decreased electrophoretic mobility of both Gro/TLE1 and Hes1. Ponceau staining is shown as a gel loading control. (c) ChIP analysis of ASCL1 promoter occupancy in HEK293 cells transfected with FLAG-tagged Hes1 alone or in combination with Pin1 and HIPK2, as indicated, using mouse anti-FLAG (lanes 2 and 6), mouse anti-HA (lanes 3 and 7) or mouse anti-glutathione S-transferase (GST) (lanes 4 and 8) antibodies (Ab), followed by PCR with primers specific for the human ASCL1 promoter. Input genomic DNA (Input) was subjected to PCR with the same primers. DNA size markers are indicated in base pairs. No ChIP product was observed if cells were transfected with FLAG vector alone or with a plasmid expressing Hes1 without a FLAG epitope, in the presence of FLAG-tagged HIPK2 and HA-tagged Pin1 (Supplementary Figure S3). (d) $\mathrm{g}-\mathrm{PCR}$ analysis of ChIP products after immunoprecipitation with antiFLAG, anti-HA or anti-GST antibodies from HEK293 cells expressing FLAG-tagged Hes1 alone or in combination with Pin1 and HIPK2. Data are expressed as fold enrichment of each ChIP experiment over ChIP values with anti-GST antibody. Bars show the mean of four replicate experiments \pm S.E.M. ( $P$-value, one-tailed Student $t$-test). (e) Electrophoretic mobility shift assays. A double-stranded oligonucleotide (probe) containing two tandem Hes1 binding sites was incubated in the absence (lane 2) or presence of nuclear extracts from either untransfected HEK293 cells (lane 3) or cells transfected with the indicated combinations of proteins (lanes 4-6). Nuclear extract from untransfected cells in the absence of oligonucleotide was loaded onto lane 1 as control (No probe). Arrow points to the position of shifted DNA:protein complex in extracts from transfected cells expressing exogenous Hes1. The position of migration of unbound probe (Free probe) is indicated

of Gro/TLE1:Hes1 to DNA. Interestingly, electrophoretic mobility shift assays have shown that the expression of Pin1 and HIPK2 abolishes DNA binding by Hes1, suggesting that Hes 1 could be directly modified by HIPK2 or Pin1 (or both). It should be mentioned, however, that because the cell lysates used in these experiments contained endogenous Gro/TLE, the observed effect might have been mediated by posttranslational modifications of Gro/TLE present in a complex with Hes1.

The present studies have shown further that the negative effect of Pin1 and HIPK2 on the transcriptional repression activity of the Gro/TLE1:Hes1 complex is biologically relevant in the context of cortical neuronal differentiation. Gro/TLE1, Hes1, Pin1 and HIPK2 are expressed in undifferentiated cortical neural progenitor cells during the neurogenic phase. Endogenous Gro/ TLE1 and Pin1 form a complex in the mouse cerebral cortex during active neuronal differentiation, when mechanisms must be in place to limit the anti-neurogenic function of Gro/TLE1 and Hes1. Pharmacological inhibition, or shRNA-mediated silencing, of Pin1 activity in cortical progenitor cells causes decreased neuronal differentiation and increased numbers of cells expressing markers of the undifferentiated neural progenitor state, in 
a
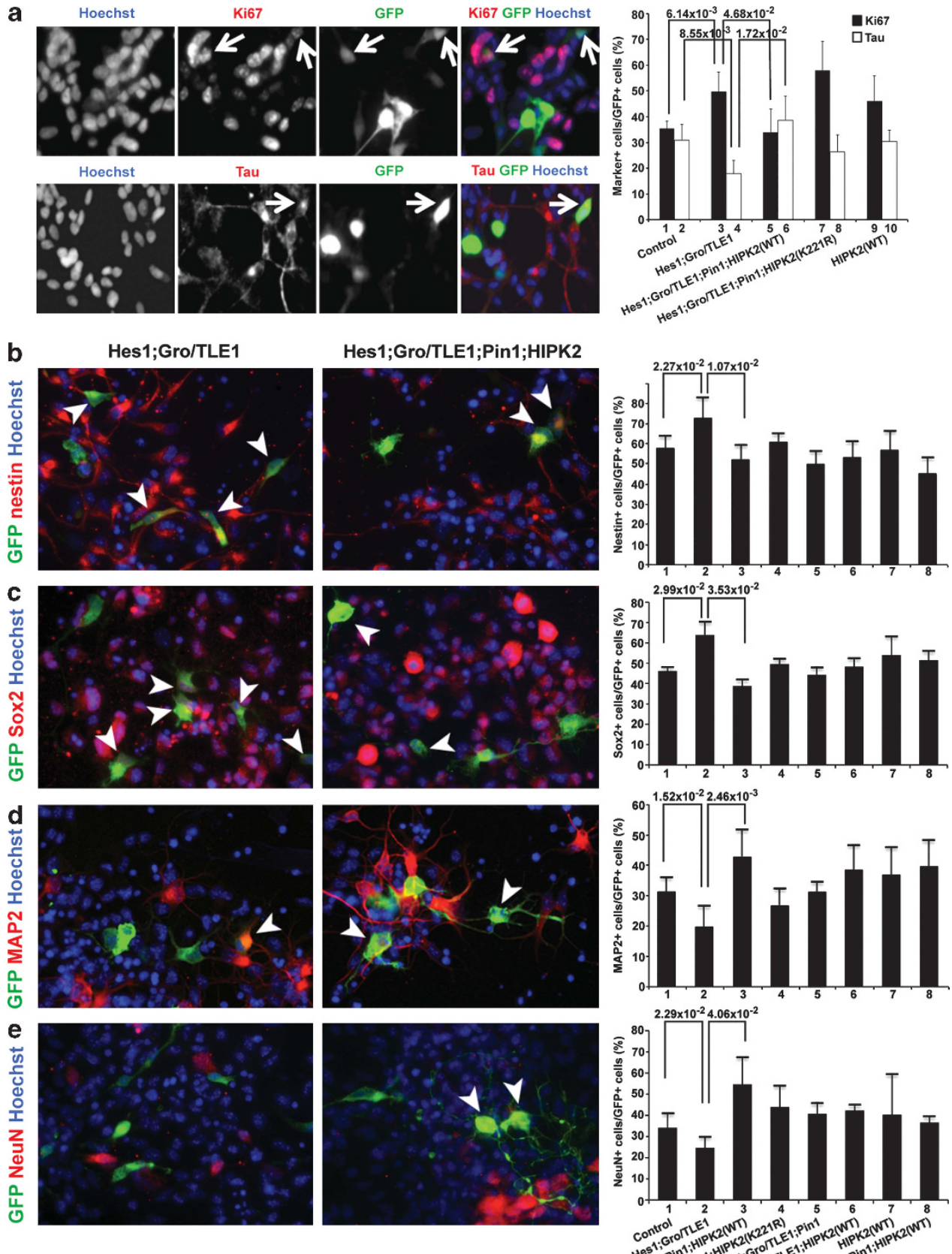

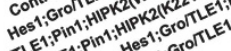

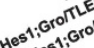

Figure 6 Inhibition of the anti-neurogenic function of Gro/TLE1:Hes1 complex by HIPK2 and Pin1. (a) SH-SY5Y neuroblastoma cells induced to undergo neuronal differentiation by treatment with retinoic acid were transfected with GFP alone (Control) or together with the combinations of proteins indicated in right-hand panel, followed by quantification of the fractions of GFP ${ }^{+}$cells coexpressing Ki67 or Tau $72 \mathrm{~h}$ after induction. Double-label immunofluorescence analysis of the expression of GFP and either Ki67 or Tau is shown in left-hand panel. Arrows point to examples of double-labeled cells. Quantification of the fraction of GFP ${ }^{+} \mathrm{SH}_{-} \mathrm{SY} 5 \mathrm{Y}$ cells coexpressing Ki67 or Tau is shown in right-hand panel (mean \pm S.D. of five separate experiments performed in duplicates; $P$-values, two-tailed Student $t$-test). Pin1 and HIPK2(WT), but not HIPK2(K221R), block the decrease in Tau ${ }^{+}$neurons and increase in Ki67 ${ }^{+}$mitotic cells caused by Gro/TLE1 and Hes1. (b-e) Primary cultures of E13.5 mouse cortical neural progenitor cells were transfected with GFP alone (Control) or together with the combinations of proteins indicated in right-hand panels, followed by double-label immunofluorescence analysis of the expression of GFP and either nestin (b), Sox2 (c), MAP2 (d) or NeuN (e) (arrowheads point to examples of double-labeled cells). Additional double-labeling immunofluorescence data can be found in Supplementary Figure S4. Graphs depicting the quantification of the fractions of GFP ${ }^{+}$cells coexpressing the indicated markers $72 \mathrm{~h}$ after transfection are shown in right-hand panels (mean \pm S.D. of five separate experiments performed in duplicates; $P$-values, two-tailed Student $t$-test). Pin1 and HIPK2(WT), but not HIPK2(K221R), block the decrease in neuronal cells and increase in neural progenitor cells caused by Gro/TLE1 and Hes1 
a

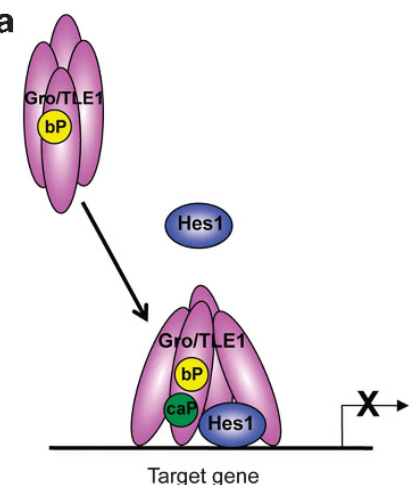

b

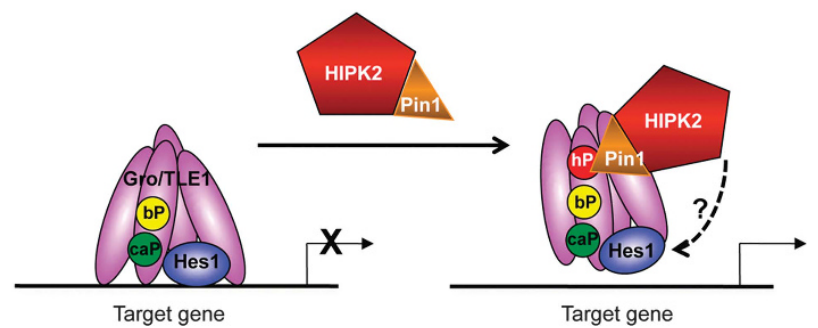

Figure 7 Proposed model of regulation of Gro/TLE1:Hes1 complex. (a) Gro/TLE1 is a basally phosphorylated ('bP'; yellow circle) protein ${ }^{18}$ that lacks DNA-binding activity but becomes recruited to specific target genes through association with Hes1. The interaction with Hes1 leads to cofactor-activated phosphorylation ('caP'; green circle) of Gro/TLE1, which results in increased affinity of Gro/TLE1 for chromatin components and optimal transcriptional repression activity. ${ }^{11,15,18}$ Binding of Hes1 also induces changes in Gro/TLE1 conformation that expose previously unavailable phosphorylation sites. (b) The Gro/TLE1:Hes1 complex is recognized by the HIPK2:Pin1 complex in a manner that depends on the binding of Hes1 to Gro/TLE1. This event results in HIPK2-mediated hyperphosphorylation ('hP'; red circle) of Gro/TLE1 creating new phosphorylated Ser/Thr-Pro site(s) that are bound by Pin1. HIPK2 may also mediate phosphorylation of Hes1 but this possibility remains to be determined. HIPK2-mediated hyperphosphorylation and/or Pin1-mediated isomerization of specific Ser/Thr-Pro site(s) weaken the interaction of the Gro/TLE1:Hes1 complex with chromatin, thereby inhibiting Gro/TLE1:Hes1-mediated transcriptional repression

agreement with recent studies showing that Pin1 knockout in mice decreases neuronal differentiation in cultured neural progenitor cells. ${ }^{25}$ More importantly, we have shown that expression of Pin1 and HIPK2 blocks the ability of Gro/ TLE1:Hes1 to inhibit neuronal differentiation in both retinoic acid-treated SH-SY5Y neuroblastoma cells and primary cultures of cortical neural progenitor cells.

In summary, the present studies provide evidence that Pin1 and HIPK2 form a complex important for the regulation of cortical neurogenesis. We propose that these enzymes act, at least in part, to mediate post-translational modifications that impair the transcriptional repression activity of Gro/TLE1:Hes1, thereby antagonizing the anti-neurogenic function of these proteins in the developing cerebral cortex. In this model, Hes1 binding sets in motion a Gro/TLE1 'phosphorylation crescendo' that first ensures that the Gro/ TLE1:Hes1 complex is recruited to specific DNA sites in a transcriptional repression-competent state and then predisposes this complex to subsequent Pin1:HIPK2-mediated post-translational modifications (Figure 7). These events act as either positive or negative switches, respectively, in the regulation of the biological activity of Gro/TLE1:Hes1 during cortical neurogenesis.

\section{Materials and Methods}

DNA plasmids. The following expression and reporter plasmids were used: pCMV2-FLAG-Gro/TLE1, ${ }^{11}$ pCMV2-FLAG-Gro/TLE1(S239A), ${ }^{11}$ pCMV2FLAG-Gro/TLE1(S286A), ${ }^{15}$ pCMV2-FLAG-Gro/TLE1(S286E), ${ }^{15}$ pCMV2-FLAGGro/TLE1(S289A), ${ }^{15}$ pCMV2-FLAG-Gro/TLE1(S298A), ${ }^{15}$ pCMV2-FLAG-Gro/ TLE1 $\triangle Q,{ }^{32}$ pCMV2-FLAG-Hes1, ${ }^{13}$ pCMV2-FLAG-Hes1 $\left(\triangle\right.$ WRPW), ${ }^{13}$ pRc-CMVHes1 ${ }^{15}$ pCMV2-FLAG-Runx $1,{ }^{36}$ pFLAG-HIPK2(WT), ${ }^{31}$ pFLAG-HIPK2(K221R), ${ }^{31}$ pcDNA3-Pin1-HA, ${ }^{31}$ pcDNA3-Pin1(S67E)-HA, ${ }^{31}$ pEGFP, ${ }^{11,12,37}$ pFOX-Neurogenin3-promoter-luciferase ${ }^{37}$ and pRSV- $\beta$-galactosidase. ${ }^{15}$

Animal procedures and immunofluorescence analysis of frozen sections. Animal procedures were conducted in accordance with the guidelines of the Canadian Council for Animal Care and were approved by the Montreal Neurological Institute Animal Care Committee. For staging of mouse embryos, the day of the appearance of the vaginal plug was considered as E0.5. E13.5 CD1 mouse embryos (Charles River Canada, Saint Constant, QC, Canada) were recovered and fixed with $4 \%$ paraformaldehyde in phosphate-buffered saline. After fixation, brains were cryoprotected by immersion in 30\% sucrose, frozenembedded in Tissue-Tek O.C.T. compound (Sakura Finetek USA, Torrance, CA, USA) and stored at $-80^{\circ} \mathrm{C}$. Frozen tissues were cryostat sectioned at $20 \mu \mathrm{m}$ and mounted onto SuperFrost Plus slides (Fisher Scientific, Ottawa, ON, Canada). Coronal sections through E13.5 mouse brains were rinsed twice in HEPESbuffered saline and then preincubated for $1 \mathrm{~h}$ in blocking solution, which was provided by the 'mouse-on-mouse' (MOM) kit purchased from Vector Laboratories Inc (Burlingame, CA, USA). Sections were then incubated for $1-2 \mathrm{~h}$ at room temperature in blocking solution containing rat anti-Gro/TLE $E^{11,12,32,38}$ (i.e., 'panTLE'; $1: 20)$ and rabbit anti-Pin $1^{39}(1: 500)$ antibodies.

Cell line culture, transfection, lysis and phosphatase treatment. Human embryonic kidney HEK293 cells were cultured, transfected using the SuperFect reagent and subjected to cell lysis as described. ${ }^{11-13}$ Treatment of cell lysates with calf intestinal phosphatase was performed as described. ${ }^{23}$

Coimmunoprecipitation. For immunoprecipitation of endogenous proteins, dissected alar telencephalon from E13.5 CD1 mouse embryos was rinsed in ice-cold Hank's balanced salt solution and then incubated in 10 packed-tissue volumes of lysis buffer $(25 \mathrm{mM}$ Tris/ $/ \mathrm{HCl}, \mathrm{pH} 7.8,150 \mathrm{mM} \mathrm{NaCl}, 1 \mathrm{mM}$ EDTA, $1 \%$ Triton X-100) supplemented with 'Complete' protease inhibitor cocktail (Roche Applied Science, Laval, QC, Canada), and $1 \mathrm{mM}$ PMSF. Tissue was mechanically triturated and incubated for $15 \mathrm{~min}$ on ice, followed by centrifugation at $14000 \times \mathrm{g}$ for $15 \mathrm{~min}$. The supernatant was recovered and subjected to immunoprecipitation of endogenous Gro/TLE1 using rabbit anti-Gro/TLE1 $1^{10,12,23}$ or control rabbit serum using protein A/G-Plus agarose beads (Santa Cruz Biotechnology, Santa Cruz, CA, USA). Collected and thoroughly rinsed immunoprecipitates were resuspended in $60 \mathrm{mM} \mathrm{Tris} / \mathrm{HCl}, \mathrm{pH} 6.8,10 \%$ glycerol and $2 \%$ SDS, followed by incubation at room temperature for $10 \mathrm{~min}$ and recovery of the eluted material by quick centrifugation. Eluates, together with $1 / 10$ of input lysate, were incubated in the presence of $100 \mathrm{mM}$ dithiothreitol, followed by SDS-polyacrylamide gel electrophoresis, transfer to nitrocellulose and western blotting with mouse anti-Pin1 (1:2000; R\&D Systems, Minneapolis, MN, USA) and rabbit anti-Gro/TLE1 $(1 ; 1000)$ antibodies. For immunoprecipitation of epitope-tagged proteins, HEK293 cells were transfected with plasmids pCMV2-FLAG-Gro/TLE1 (200 ng/transfection) and pCMV2-FLAG-Hes1 (or pCMV2-FLAG-Hes1( $\triangle W R P W)$ (200 ng/transfection) in the absence or presence of pcDNA3-Pin1-HA (or pcDNA3-Pin1(S67E)-HA) (400 ng/transfection) and pFLAG-HIPK2(WT) (or pFLAG-HIPK2(K221R)) (400 ng/ transfection), followed by preparation of cell lysates and immunoprecipitation with either rabbit anti-Gro/TLE1, mouse anti-HA (Covance, Emeryville, CA, USA) or preimmune serum as described. ${ }^{11,12,15}$ Immunoprecipitates were analyzed 
together with each input lysate collected before immunoprecipitation by western blotting using mouse anti-FLAG (1:10 000; Sigma-Aldrich, St. Louis, MO, USA), mouse anti-HA $(1: 5000)$ or rat anti-Gro/TLE $(1: 20)$ antibodies.

Biochemical fractionation. Post-nuclear supernatants and chromatinenriched fractions were obtained as described previously. ${ }^{17}$ Subcellular fractions were analyzed by western blotting using anti-FLAG, anti-Gro/TLE or anti-HA antibodies.

Transient transfection/transcription assays. HEK293 cells were transfected using the SuperFect reagent. In all cases, the total amount of transfected DNA was adjusted at $3.0 \mu \mathrm{g}$ per well using empty $p c D N A 3$ plasmid. Cells were transfected with the $p F O X$-Neurogenin3-promoter-luciferase construct $(1.0 \mu \mathrm{g} /$ transfection) alone or together with pCMV2-FLAG-Hes1 $(50 \mathrm{ng} /$ transfection) in the absence or presence of PCMV2-FLAG-Gro/TLE1 (100 ng/transfection), pCMV2-FLAG-Gro/TLE1 1 Q (200 ng/transfection), pcDNA3-Pin1-HA (400 ng/transfection), pFLAG-HIPK2(WT) (400 ng/transfection) or pFLAG-HIPK2(K221R) $(400 \mathrm{ng} /$ transfection), as indicated in the appropriate figure legend. In each case, $250 \mathrm{ng} /$ transfection of $\beta$-galactosidase expression plasmid, $p R S V$ - $\beta g a l$, was used to normalize for transfection efficiency. Twenty-four hours after transfection, cells were subjected to determination of luciferase activity as described. ${ }^{11} 13$

Chromatin immunoprecipitation. ChIP experiments were performed using the Magna ChIP G chromatin immunoprecipitation kit (Millipore, Billerica, MA, USA). All steps were performed according to the manufacturer's instructions. HEK293 cells were transfected with PCMV2-FLAG-Hes1 (25 ng/transfection) alone or together with pFLAG-HIPK2 (100 ng/transfection) and pcDNA3-Pin1-HA (100 ng/transfection), followed by cross-linking for $10 \mathrm{~min}$ using formaldehyde, sonication to yield 200-500 bp fragments and immunoprecipitation with either antiFLAG antibody or the following control, irrelevant, antibodies: anti-HA and mouse anti-glutathione S-transferase (Santa Cruz Biotechnology). Chromatin:antibody complexes were harvested using protein $\mathrm{G}$ magnetic beads and a magnetic separator, washed extensively and incubated at $62{ }^{\circ} \mathrm{C}$ in the presence of 'ChIP Elution Buffer' containing $0.1 \mathrm{mg} / \mathrm{ml}$ proteinase $\mathrm{K}$ to reverse the cross-linking and to recover the DNA. Immunoprecipitated DNA was purified using spin columns and reagents supplied with the Magna ChIP G kit and analyzed for the human ASCL1 promoter sequence by $\mathrm{PCR}$ using oligonucleotide primers whose respective $5^{\prime}$ ends were located $546 \mathrm{bp}$ (primer ASCL1p-F) and $170 \mathrm{bp}$ (primer ASCL1p-R) upstream from the starting ATG codon, resulting in a product of $376 \mathrm{bp}$. The amplified region contains two tandem copies 5'-CACGCGAGCGCCACGCG- $3^{\prime}$ of the N-box, a canonical Hes binding site. These sequences correspond to the two previously described tandem Hes1-binding sites in the proximal region of the rat Ascl1 gene. ${ }^{14}$ The sequence of the oligonucleotide primers was as follows: ASCL1p-F, 5'-ACGTGGAAGGCGCACCGACAG-3'; ASCL1p- $R, 5^{\prime}$-GAGACTTCT TAGAGTGGGAGCA-3'.

Quantitative PCR. Products of ChIP experiments were analyzed by q-PCR using the CFX96 Touch Real-Time PCR Detection System using SsoFast EvaGreen Supermix (Bio-Rad Laboratories, Hercules, CA, USA). The following oligonucleotides were used: ASCL1p-F2: 5'-GCAGCCTTAGTAGGAGAGGAA-3'; ASCL1p-R2: 5'-AAAGCAGAAGCAGCAGCA-3'. Values were calculated as fold enrichment of each condition over the ChIP data obtained using the anti-GST antibody as control.

Electrophoretic mobility shift assay. Reactions were performed in a volume of $20 \mu \mathrm{l}$ containing a gel shift binding buffer provided by the 'LightShift Chemiluminescent EMSA kit' (Pierce Biotechnology, Rockford, IL, USA), $50 \mathrm{ng} / \mu$ l poly-(dl:dC) and $4 \mu \mathrm{g}$ of nuclear extracts. A double-stranded oligonucleotide containing two tandem $\mathrm{N}$-box sequences (sense strand: $5^{\prime}$-CTAGACGCCAC GAGCCACAAGGATTG-3'; antisense strand: $5^{\prime}$-CTAGCAATCCTTGTGGCTCGT $\overline{\mathrm{GGC}} \mathrm{GT}-3^{\prime}$ ) was labeled at $3^{\prime}$-ends with biotin (Integrated DNA Technologies, Toronto, ON, Canada) and used as a probe $(\sim 10 \mathrm{fmol})$. Samples were incubated for $20 \mathrm{~min}$ at $22{ }^{\circ} \mathrm{C}$ before electrophoresis on $5 \%$ polyacrylamide gels $(29: 1$ acrylamide:bis-acrylamide) in $0.5 \mathrm{X}$ TBE buffer.

Retinoic acid-induced neuronal differentiation of neuroblastoma cells. Human neuroblastoma SH-SY5Y cells ${ }^{34,35,40}$ were cultured and transfected with plasmids encoding GFP and the combinations of proteins indicated in the appropriate figure legend using the HD Fugene reagent (Roche Applied Science). Neuronal differentiation was induced by the treatment of SH-SY5Y cells with $10 \mathrm{mM}$ retinoic acid for 3 days. After this time, cells were subjected to doublelabel immunofluorescence analysis of the expression of GFP and either Ki67 or Tau using the following antibodies: mouse anti-Ki67 (1:500; BD Pharmingen, San Diego, CA, USA) or mouse anti-Tau (1:100; Millipore).

Primary cultures of cortical neural progenitor cells. Primary neural progenitor cells from E13.5 mouse neocortices were obtained and cultured as described. ${ }^{11,12,37}$ Double-label immunofluorescence analysis of endogenous proteins was performed as described ${ }^{11,41}$ using the following primary antibodies: rat anti-Gro/TLE (1:10), rabbit anti-Pin1 (1:500), mouse anti-Pin1 (1:200; R\&D Systems) or rabbit anti-HIPK2 ${ }^{42}(1: 300)$. For differentiation assays, cells were transfected as described ${ }^{11,12,37}$ after $48 \mathrm{~h}$ in vitro using the following plasmids in the combinations indicated in the appropriate figure legend: $p E G F P(0.1 \mu \mathrm{g} / \mathrm{well})$ alone or in combination with pCMV2-FLAG-Gro/TLE1 (0.4 $\mu \mathrm{g} /$ well), pCMV2-FLAGHes1 $(0.4 \mu \mathrm{g} / \mathrm{well})$, pFLAG-HIPK2 or pFLAG-HIPK2(K221R) $(0.4 \mu \mathrm{g} / \mathrm{well})$ and pcDNA3-Pin1-HA (0.4 $\mu \mathrm{g} / \mathrm{well})$. The pEGFP plasmid was always present at a $1: 4$ ratio compared with the effector plasmids, a strategy that results in greater than $90 \%$ of transfected cells coexpressing GFP and the proteins of interest. ${ }^{11,12,15,37}$ Three days following transfection, cells were analyzed by immunofluorescence using antibodies against Ki67 (1:500; BD Pharmingen), nestin (1:100; Millipore), Sox2 (1:250; R\&D Systems), type III $\beta$-tubulin (1:1000; Promega, Madison, WI, USA), MAP2 (1:1000; Sigma-Aldrich) and NeuN (1:250; Millipore), as described. ${ }^{41,43}$ Cells were counterstained with Hoechst 33258 (Sigma-Aldrich) before examination by fluorescence microscopy. Grayscale images were digitally assigned to the appropriate red or green channel using Northern Eclipse software (Empix Imaging, Inc., Mississauga, ON, Canada). Three to six random fields of each condition (per experiment) were used for quantitation of the percent of GFP-positive cells coexpressing specific markers. Fields were photographed using a $\times 10$ objective, with an average of about 300 cells per field. Cells were identified manually in Photoshop (Adobe Systems, San Jose, CA, USA) and quantitated using Image $\mathrm{J}$ software (National Institutes of Health, Bethesda, MD, USA)

Pharmacological inhibition of Pin1. Cortical progenitor cells were established from E13.5 CD1 embryos and plated at a density of $3 \times 10^{5} \mathrm{cells} / \mathrm{ml}$. Prior to treatment with the Pin1 inhibitor juglone, half of the culture medium was removed on day in vitro (DIV) 2, kept as conditioned medium, and replaced with fresh medium together with $5 \mu \mathrm{M}$ juglone (Sigma-Aldrich) dissolved in DMSO, or DMSO alone. Six hours later, the medium was replaced with a 1:1 mix of fresh and conditioned medium. On DIV 5, cells were either fixed and subjected to immunocytochemical analysis or lysed for Western blot studies. Immunocytochemistry was performed using antibodies against Ki67, nestin, MAP2, or type III $\beta$-III-tubulin. For each condition and each antibody, 5 different fields were photographed using a 20X objective, with an average of about 300 cells per field. Cell counting was performed as described above. Western blot studies were conducted using the following antibodies: anti-phospho-histone H3 (1:2000; Millipore), anti-type III $\beta$-III-tubulin (1:10000), anti-GAP-43 (1:2500; Millipore), and anti- $\beta$-actin (1: 10000 ; Abcam Inc., Toronto, ON, Canada).

Pin1 knockdown. Cortical progenitor cells were plated at a density of $2 \times 10^{5} \mathrm{cells} / \mathrm{ml}$. On DIV 2, half of the medium was removed and cells were transduced with lentivirus expressing either non-silencing shRNA (Sigma-Aldrich, Cat No. SGC202V) or Pin1 shRNA (Sigma-Aldrich, Cat No. TRCN0000321124), at a multiplicity of infection of 5 . Five hours later, a volume of fresh medium equal to the volume of medium removed before transduction was added. On DIV 5 and 7 , half of the medium was replaced with fresh medium. On DIV 8, cells were lysed and subjected to western blot analysis using antibodies against Pin1, phosphohistone $\mathrm{H} 3$, type III $\beta$-III-tubulin, GAP-43 and $\beta$-actin.

Statistical analysis. Statistical comparisons were made using two-tailed Student's t-test. All statistical tests were performed with GraphPad Prism software (GraphPad, La Jolla, CA, USA). Significance levels was set at $P<0.05$. Where appropriate, additional statistical details are provided in the figure legends.

\section{Conflict of Interest}

The authors declare no conflict of interest. 
Acknowledgements. We thank Dr. Thomas Hofmann (German Cancer Research Centre) for anti-HIPK2 antibody and Dr. Morena Zusso and Edwige Barajas for their help during these studies. LM and RD were supported by doctoral studentships from the FRQS and Vanier Canada CGS, respectively. RR was supported by grants from Associazione Italiana per la Ricerca sul Cancro (AIRC IG no. 10338) and Italian Ministry of Health (Ricerca Corrente). GDS was funded by AIRC, Italian University and Research Ministry (PRIN and FIRB grants), and FVG (regional grant AITT). SS was supported by grants from the Canadian Institutes of Health Research (MOP-13957 and MOP-42479). SS is a James McGill Professor of McGill University.

1. Hsieh J. Orchestrating transcriptional control of adult neurogenesis. Genes Dev 2012; 26: 1010-1021.

2. Martynoga $B$, Drechsel $D$, Guillemot $F$. Molecular control of neurogenesis: a view from the mammalian cerebral cortex. Cold Spring Harb Perspect Biol 2012; 4: pii: a008359.

3. Ishibashi M, Moriyoshi K, Sasai Y, Shiota K, Nakanishi S, Kageyama R. Persistent expression of helix-loop-helix factor HES-1 prevents mammalian neural differentiation in the central nervous system. EMBO J 1994; 13: 1799-1805.

4. Ishibashi M, Ang SL, Shiota K, Nakanishi S, Kageyama R, Guillemot F. Targeted disruption of mammalian hairy and Enhancer of split homolog-1 (HES-1) leads to up-regulation of neural helix-loop-helix factors, premature neurogenesis, and severe neural tube defects. Genes Dev 1995; 9: 3136-3148.

5. Kageyama R, Ohtsuka T, Shimojo H, Imayoshi I. Dynamic Notch signaling in neural progenitor cells and a revised view of lateral inhibition. Nat. Neurosci 2008; 11: 1247-1251

6. Paroush Z, Finley RL, Kidd T, Wainwright SM, Ingham PW, Brent R et al. Groucho is required for Drosophila neurogenesis, segmentation, and sex determination and interacts directly with hairy-related proteins. Cell 1994; 79: 805-815.

7. Fisher AL, Ohsako S, Caudy M. The WRPW motif of the hairy-related basic helix-loop-helix repressor proteins acts as a 4-amino-acid transcription repression and protein-protein interaction domain. Mol Cell Biol 1996; 16: 2670-2677.

8. Grbavec D, Lo R, Liu Y, Stifani S. Transducin-like Enhancer of split 2, a mammalian homologue of Drosophila Groucho, acts as a transcriptional repressor, interacts with Hairy/Enhancer of split proteins, and is expressed during neuronal development. Eur J Biochem 1998; 258: 339-349.

9. Dehni G, Liu Y, Husain J, Stifani S. TLE expression correlates with mouse embryonic segmentation, neurogenesis, and epithelial determination. Mech Dev 1995; 53: 369-381.

10. Yao J, Liu Y, Lo R, Tretjakoff I, Peterson A, Stifani S. Disrupted development of the cerebral hemispheres in transgenic mice expressing the mammalian Groucho homologues Transducin-like Enhancer of split 1 in postmitotic neurons. Mech Dev 2000; 93: 105-115.

11. Nuthall HN, Joachim K, Stifani S. Phosphorylation of serine 239 of Groucho/TLE1 by protein kinase CK2 is important for inhibition of neuronal differentiation. Mol Cell Biol 2004; 24: 8395-8407.

12. Buscarlet M, Perin A, Laing A, Brickman JM, Stifani S. Inhibition of cortical neuron differentiation by Groucho/TLE1 requires interaction with WRPW, but not Eh1, repressor peptides. J Biol Chem 2008; 283: 24881-24888.

13. McLarren KW, Theriault FM, Stifani S. Association with the nuclear matrix and interaction with Groucho and RUNX proteins regulate the transcription repression ability of the basic helix loop helix factor Hes1. J Biol Chem 2001; 276: 1578-1584.

14. Ju BG, Solum D, Song EJ, Lee KJ, Rose DW, Glass CK et al. Activating the PARP-1 sensor component of the Groucho/TLE1 corepressor complex mediates a CaMKinase III-dependent neurogenic gene activation pathway. Cell 2004; 119: 815-829.

15. Buscarlet M, Hermann R, Lo R, Tang Y, Joachim K, Stifani S. Cofactor-activated phosphorylation is required for inhibition of cortical neuron differentiation by Groucho/TLE1. PLoS One 2009; 4: e8107.

16. Jennings BH, Pickles LM, Wainwright SM, Roe SM, Pearl LH, Ish-Horowicz D. Molecular recognition of transcriptional repressor motifs by the WD domain of the Groucho/TLE corepressor. Mol Cell 2006; 22: 645-655.

17. Palaparti A, Baratz A, Stifani S. The Groucho/transducin-like enhancer of split transcriptional repressors interact with the genetically defined amino-terminal silencing domain of histone H3. J Biol Chem 1997; 272: 26604-26610.

18. Nuthall HN, Husain J, McLarren KW, Stifani S. Role for Hes1-induced phosphorylation in Groucho-mediated transcriptional repression. Mol Cell Biol 2002; 22: 389-399.

19. Liou YC, Zhou XZ, Lu KP. Prolyl isomerase Pin1 as a molecular switch to determine the fate of phosphoproteins. Trends Biochem Sci 2011; 36: 501-514.
20. Theuerkorn M, Fischer G, Schiene-Fischer C. Prolyl cis/trans isomerase signalling pathways in cancer. Curr Opin Pharmacol 2011; 11: 281-287.

21. Rinaldo C, Prodosmo A, Siepi F, Soddu S. HIPK2: a multitalented partner for transcription factors in DNA damage response and development. Biochem Cell Biol 2007; 85: 411-418.

22. Sombroek D, Hofmann TG. How cells switch HIPK2 on and off. Cell Death Differ 2009; 16 : 187-194.

23. Husain J, Lo R, Grbavec D, Stifani S. Affinity for the nuclear compartment and expression during cell differentiation implicate phosphorylated Groucho/TLE1 forms of higher molecular mass in nuclear functions. Biochem $J$ 1996; 317: 523-531.

24. Fila C, Metz C, van der Sluijs P. Juglone inactivates cysteine-rich proteins required for progression through mitosis. J Biol Chem 2008; 283: 21714-21724.

25. Nakamura K, Kosugi I, Lee DY, Hafner A, Sinclair DA, Ryo A et al. Prolyl isomerase Pin1 regulates neuronal differentiation via $\beta$-catenin. Mol Cell Biol 2012; 32: 2966-2978.

26. Choi CY, Kim YH, Kim YO, Park SJ, Kim EA, Riemenschneider W et al. Phosphorylation by the DHIPK2 protein kinase modulates the corepressor activity of Groucho. J Biol Chem 2005; 280: 21427-21436.

27. Lee W, Andrews BC, Faust M, Walldorf U, Verheyen E. Hipk is an essential protein that promotes Notch signal transduction in the Drosophila eye by inhibition of the global co-repressor Groucho. Dev Biol 2009; 325: 263-272.

28. Sung KS, Go YY, Ahn JH, Kim YH, Kim Y, Choi CY. Differential interactions of the homeodomain-interacting protein kinase 2 (HIPK2) by phosphorylation-dependent sumoylation. FEBS Lett 2005; 579: 3001-3008.

29. Buscarlet M, Stifani S. The 'Marx' of Groucho on development and disease. Trends Cell Biol 2007; 17: 353-361.

30. Jennings BH, Ish-Horowicz D. The Groucho/TLE/Grg family of transcriptional co-repressors. Genome Biol 2008: 9: 205-211.

31. Sorrentino G, Mioni M, Giorgi C, Ruggeri N, Pinton P, Moll U et al. The prolyl-isomerase Pin1 activates the mitochondrial death program of p53. Cell Death Differ 2013; 20: 198-208.

32. Todd KJ, Lan-Chow-Wing N, Salin-Cantegrel A, Cotter A, Zagami C, Lo R et al. Establishment of motor neuron- $\mathrm{V} 3$ interneuron progenitor domain boundary in ventral spinal cord requires Groucho-mediated transcriptional corepression. PLoS One 2012; 7: e31176.

33. Påhlman S, Ruusala Al, Abrahamsson L, Mattsson ME, Esscher T. Retinoic acid-induced differentiation of cultured human neuroblastoma cells: a comparison with phorbolesterinduced differentiation. Cell Differ 1984; 14: 135-144.

34. Encinas M, Iglesias M, Liu Y, Wang H, Muhaisen A, Cena V et al. Sequential treatment of SH-SY5Y cells with retinoic acid and brain-derived neurotrophic factor gives rise to fully differentiated, neurotrophic factor-dependent, human neuron-like cells. J Neurochem 2000; 75: 991-1003.

35. Megiorni F, Mora B, Indovina P, Mazzilli MC. Expression of neuronal markers during NTera2/cloneD1 differentiation by cell aggregation method. Neurosci Lett 2005; 373: 105-109.

36. McLarren KW, Lo R, Grbavec D, Thirunavukkarasu K, Karsenty G, Stifani S. The mammalian basic helix loop helix protein Hes 1 binds to and modulates the transactivating function of the Runt-related factor Cbfa1. J Biol Chem 2000; 275: 530-538.

37. Gratton MO, Torban E, Belanger-Jasmin S, Theriault F, German MS, Stifani S. Hes6 promotes cortical neurogenesis and inhibits Hes1 transcription repression activity by multiple mechanisms. Mol Cell Biol 2003; 23: 6922-6935.

38. Stifani S, Blaumueller CM, Redhead NJ, Hill RE, Artavanis-Tsakonas S. Human homologues of a Drosophila Enhancer of split gene product define a novel family of nuclear proteins. Nat Genetics 1992; 2: 119-127.

39. Zacchi P, Gostissa M, Uchida T, Salvagno C, Avolio F, Volinia S et al. The prolyl isomerase Pin1 reveals a mechanism to control p53 functions after genotoxic insults. Nature 2002; 419: 853-857.

40. Grison A, Mantovani F, Comel A, Agostoni E, Gustinich S, Persichetti F et al. Ser46 phosphorylation and prolyl-isomerase Pin1-mediated isomerization of p53 are key events in p53-dependent apoptosis induced by mutant huntingtin. Proc Natl Acad Sci USA 2011; 108: 17979-17984.

41. Zusso M, Methot L, Lo R, Grenhalgh AD, David S, Stifani S. Regulation of postnatal forebrain amoeboid microglial cell proliferation and development by the transcription factor Runx1. J Neurosci 2012; 32: 11285-11298.

42. Hofmann TG, Möller A, Sirma H, Zentgraf H, Taya Y, Dröge W et al. Regulation of p53 activity by its interaction with homeodomain-interacting protein kinase-2. Nat Cell Biol 2002; 4: 1-10.

43. Methot L, Hermann R, Tang Y, Lo R, Al-Jehani H, Jhas S et al. Interaction and antagonistic roles of NF-kB and Hes6 in the regulation of cortical neurogenesis. Mol Cell Biol 2013; 33: 2797-2808. 